\title{
Vértigo posicional paroxístico benigno: el vértigo que todos debemos conocer
}

\author{
Benign paroxysmal positional vertigo: the vertigo that we should all know
}

\author{
Francisco José Gallardo Ollervides, ${ }^{\star}$ Laura Angélica Escalona López, ${ }^{\ddagger}$ \\ Saúl Alejandro Moreno Reynoso,§ Jaime Fernández Espinosall
}

\begin{abstract}
RESUMEN
La más frecuente causa de vértigo es el vértigo posicional paroxístico benigno, una entidad clínica que reúne varias características, y que puede y debe ser reconocida por cualquier médico, pero sobre todo por los especialistas involucrados en el estudio de los trastornos del equilibrio. En su largo y aparentemente complicado nombre, están implícitas varias de las características clínicas que lo definen por su diagnóstico. Éste, en su variedad más frecuente, es bastante sencillo, totalmente clínico, no requiere estudios de gabinete, laboratorio o imagen y su tratamiento es inmediato y altamente efectivo. En este trabajo se revisan las maniobras diagnósticas y terapéuticas más útiles para identificarlo y tratarlo adecuadamente.
\end{abstract}

Palabras clave: Vértigo posicional paroxístico benigno, maniobras diagnósticas, maniobras liberadoras.

Nivel de evidencia: III

\begin{abstract}
The most frequent cause of vertigo is benign paroxysmal positional vertigo, a clinical entity that has several characteristics that can and should be recognized by any doctor, but above all by specialists involved in the study of balance disorders. In its long, apparently complicated name, several of the clinical characteristics that define it are implicit, so its diagnosis. In its most frequent variety, is quite simple, totally clinical, it does not require cabinet, laboratory or image studies and its treatment is immediate and highly effective. In this paper we review the most useful diagnostic and therapeutic maneuvers to identify and treat it adequately.
\end{abstract}

Keywords: Benign paroxysmal positional vertigo, diagnostic maneuvers, therapeutic maneuvers.

Level of evidence: III

* Otorrinolaringólogo. Neurootólogo, Jefe de Servicio Otorrinolaringología (ORL) y Cirujano de Cabeza y Cuello (CCC), Hospital Central Militar 20082018. Ciudad de México.

‡ Otorrinolaringóloga y Cirujano de Cabeza y Cuello, práctica privada. Ciudad de México.

$\S$ Otorrinolaringólogo, adscrito al Servicio de Otorrinolaringología (ORL) y Cirugía de Cabeza y Cuello (CCC), Hospital Central Militar. Ciudad de México.

" Otorrinolaringólogo. Neurootólogo, Presidente de la Asociación Médica Centro Médico ABC. Ciudad de México.

Recibido para publicación: 26/08/2019. Aceptado: 16/12/2019.

\section{Correspondencia: Francisco José Gallardo Ollervides}

Sur 136 Núm. 116 Int. 2-A, Centro Médico ABC Observatorio Torre Sur,

Col. Las Américas, 01120, Álvaro Obregón, CDMX. Tel: 55-2699-6999

E-mail: fragallardo@yahoo.com.mx

\section{Abreviatura:}

VPPB = Vértigo posicional paroxístico benigno.

Este artículo puede ser consultado en versión completa en: www.medigraphic.com/analesmedicos

\section{INTRODUCCIÓN}

Los síntomas vestibulares y trastornos del equilibrio (vértigo, mareo, síntomas vestibulovisuales y síntomas posturales) ${ }^{1}$ son muy frecuentes y representan un verdadero reto diagnóstico, ya que su fisiopatología es compleja e involucra a varios sistemas que nos permiten tener conciencia de la posición de nuestro cuerpo en el espacio. Éstos, coordinados por el cerebelo y otras estructuras centrales, logran el milagro de la bipedestación y los movimientos complejos de desplazamiento, que representan uno de los sistemas filogenéticos más antiguos y complejos en el desarrollo del ser humano.

De todos los síntomas neurológicos, el vértigo es el que mayor impacto emocional tiene en los pacientes. ${ }^{2}$ La inseguridad, el miedo, la angustia y la ansiedad son las molestias más frecuentes, las cua- 
les están documentadas desde hace muchos años en pacientes sin antecedentes psiquiátricos y que han sufrido crisis de vértigo. ${ }^{3}$ Afortunadamente la causa más frecuente de vértigo es el vértigo posicional paroxístico benigno (VPPB), ${ }^{4,5}$ entidad clínica que reúne varias características por las cuales cualquier médico puede y debe reconocerla, pero sobre todo por los especialistas involucrados en el estudio de los trastornos del equilibrio.

En su largo y aparentemente complicado nombre, están implícitas varias de las características clínicas que lo definen, por lo que su diagnóstico, en su variedad más frecuente, ${ }^{6}$ es bastante sencillo, totalmente clínico, no requiere estudios de gabinete, laboratorio o imagen y su tratamiento es inmediato y altamente efectivo. ${ }^{7}$ Es de los pocos padecimientos en los cuales el paciente puede salir del consultorio con su problema resuelto.

\section{Epidemiología}

La incidencia del VPPB en la población general a lo largo de la vida es del $10 \%{ }^{8}$ y su índice de recurrencia puede ser de hasta $50 \% .{ }^{9}$ Es más frecuente entre la quinta y la séptima década de la vida ${ }^{10}$ y tiene una predominancia de 2:1 en el sexo femenino. ${ }^{11}$ Lamentablemente, a pesar de su frecuencia y las características mencionadas que facilitan su diagnóstico y tratamiento, muchas veces los pacientes son mal diagnosticados y en consecuencia mal orientados en su tratamiento.

\section{Cuadro clínico}

El cuadro clínico clásico consiste en una crisis de vértigo que habitualmente ocurre durante la noche en la cama, al acostarse, girarse o levantarse; esta alucinación de movimiento rotatorio dura segundos, aunque el paciente tiende a referir una mayor duración por la sensación tan desagradable e impactante que percibe cuando siente vértigo; esta crisis se repite con ciertos movimientos y en muchos casos el paciente logra identificar una postura o posición que lo desencadena, evitando conscientemente la postura que inicia la crisis. La sensación rotatoria se puede acompañar de síntomas vasovagales como palidez, diaforesis, náusea y vómito, pero no existen síntomas auditivos acompañantes (acúfeno, plenitud ótica, otalgia o hipoacusia). El paciente no refiere síntomas neurológicos como disartria, dislalia, cefalea, ni alteraciones sensitivas o motoras, pero es frecuente que manifiesten gran ansiedad y angustia por la recurrencia del síntoma.

\section{Exploración física}

Una vez obtenida la descripción sintomática anterior, debemos proceder a la búsqueda de los signos clínicos que confirman el diagnóstico, para lo cual realizaremos maniobras diagnósticas que provocan la aparición del nistagmo característico, dependiendo del oído y el canal afectado. Existen seis características del nistagmo que nos permiten confirmar el diagnóstico; las cuatro primeras se presentan al acostar o girar al paciente, una más al levantarlo o girarlo hacia el lado contrario y la última al repetir la maniobra. La identificación de las seis características del nistagmo-vértigo nos permitirá diagnosticar con toda seguridad un VPPB. Éstas son:

1. Desencadenado por el movimiento. El VPPB siempre se presentará después de que el paciente realiza un movimiento; es muy común que ocurra en la cama al girarse o al acostarse, o bien al levantar o agachar la cabeza buscando algo hacia arriba o hacia abajo.

2. Presencia de latencia. Una de las características clínicas para diferenciar un nistagmo de tipo central de uno periférico es justamente la presencia de latencia; en la mayoría de los casos de VPPB, ésta será de entre 5 y 20 segundos, aunque hay casos con latencia mínima o imperceptible y otros con latencias hasta de 30 segundos; por lo cual siempre es recomendable durante la exploración, esperar un minuto después de adoptada la posición de provocación.

3. Presencia de paroxismo. El nistagmo tiene la característica de iniciar de manera suave (in crescendo) y aumentar, llegando a un clímax máximo de velocidad (paroxismo) y luego disminuir hasta desaparecer (de crescendo).

4. Duración de segundos. En todos los casos de VPPB el vértigo y nistagmo duran segundos; la mayoría de las ocasiones entre 20 y 40 segundos y sólo en algunos casos más de 60 segundos.

5. Reversible. La dirección del nistagmo se invierte al movilizar al paciente pasando de la posición sentado a decúbito, o al girar la cabeza de izquierda a derecha y viceversa. Esto significa que, si al acostar al paciente el nistagmo bate hacia arriba y en torsión externa, al levantarlo el nistagmo batirá hacia abajo y en torsión interna; de igual manera cuando gire la cabeza hacia la izquierda, el nistagmo batirá hacia la izquierda, y cuando gire la cabeza hacia el lado contrario batirá hacia la derecha. Los estímulos excitatorios siempre son más intensos que 
los inhibitorios, por eso la intensidad del nistagmo es mayor al acostarlo que al levantarlo cuando la afección se encuentra en los canales verticales. En los canales horizontales también ocurre lo mismo, siempre es más intenso el nistagmo hacia alguno de los dos lados y corresponde al estímulo excitatorio (segunda ley de Ewald).

6. Fatigable. La intensidad del nistagmo al igual que su duración disminuyen a medida que se repiten los movimientos que lo desencadenan, de modo tal que un nistagmo muy evidente será más sutil en la segunda y aún más en la tercera repetición del movimiento que lo desencadenó.

\section{Maniobra diagnóstica para vértigo posicional paroxístico benigno de canales verticales}

La maniobra clásica para diagnóstico de VPPB de canal posterior es la descrita en 1952 por Dix y Hallpike, ${ }^{12}$ la cual consiste en colocar al paciente de manera longitudinal en la mesa de exploración con las piernas extendidas y el torso elevado, girar la cabeza 45 grados hacia la izquierda o derecha según sea el oído a explorar y acostarlo de modo tal que la cabeza quede ligeramente extendida $\left(30^{\circ}\right.$ aproximadamente) al alcanzar la posición de decúbito dorsal. Se debe explicar al paciente el movimiento antes de realizarlo y solicitarle que mantenga los ojos abiertos para poder observar las características del nistagmo, pero pudiendo parpadear. Se debe mantener esta posición por lo menos 45 segundos, ya que hay pacientes que tienen latencias de hasta 35 segundos (Figura 1).

Cuando se realiza una maniobra de Dix-Hallpike, debido a su situación ortogonal, se estimulan un par de canales que están en el mismo eje funcional, es decir, el posterior del lado hacia donde se realiza la maniobra y el anterior del lado contralateral (Figura 2). Como el VPPB de canal anterior es extremadamente raro (1-2\%), para efectos prácticos, al realizar esta

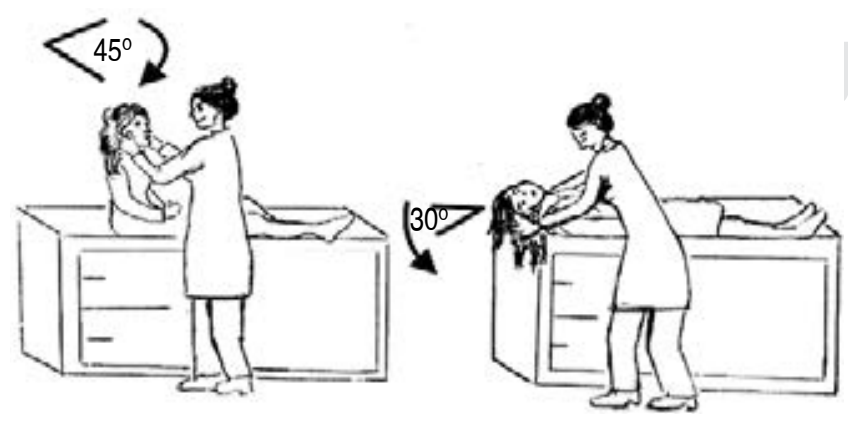

Figura 1: Maniobra diagnóstica para canales verticales de Dix-Hallpike.

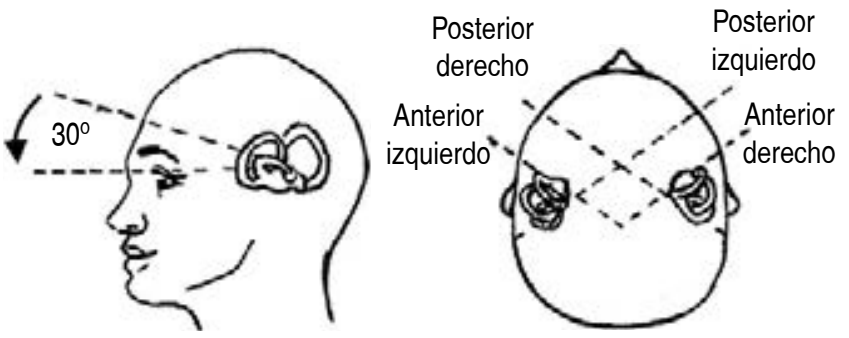

Figura 2: Disposición ortogonal de los canales semicirculares.

maniobra y observar un nistagmo de canal posterior sabremos cuál es el canal enfermo simplemente observando hacia qué lado hubo nistagmo y ése será el oído enfermo. Si se presenta nistagmo al realizar la maniobra hacia la izquierda y también hacia la derecha, entonces tendremos afección bilateral. En caso de que la dirección del nistagmo corresponda a un estímulo del canal anterior (mixto: hacia abajo y torsión interna) tendríamos uno de los raros casos de VPPB del canal anterior ${ }^{13}$ contralateral a la maniobra. Esto sólo aplica para los canales verticales, ya que los horizontales por su orientación y eje funcional tienen ciertas peculiaridades que comentaremos más adelante.

Maniobra diagnóstica para vértigo posicional paroxístico benigno de canales horizontales

Para el diagnóstico del VPPB de canal lateral existen tres maniobras: la maniobra de estímulo mínimo, la maniobra de giro o rotación lateral (roll test) y la maniobra de acostado lateral (side-lying).

La maniobra de estímulo mínimo o flexión de la cabeza $^{14}$ se realiza con el paciente sentado inclinando la cabeza hacia adelante-abajo y en seguida hacia atrás-arriba y observar la respuesta ocular en cada posición (Figura 3).

La maniobra de giro lateral (roll test o PagniniMcClure $)^{15}$ puede realizarse con el paciente en decúbito dorsal o estando sentado; la cabeza debe inclinarse hacia adelante 30 grados para colocar los canales semicirculares laterales en posición completamente horizontal, o estando acostado el paciente debe levantar la cabeza 30 grados y realizar los giros que, si la movilidad del paciente lo permite, deben ser de más de 45 grados hacia un lado; se debe esperar la respuesta de nistagmo y luego regresar la cabeza a la posición central y girar para el otro lado (Figura 4). Al realizar la prueba en decúbito supino se suman dos fuerzas: la de gravedad y la inercial por el movimiento; en cambio al realizar la prueba con el paciente sentado sólo se 
usa la inercia del movimiento cefálico en el plano yaw, el cual el explorador puede controlar modificando la fuerza y velocidad del giro, lo que podría ser deseable en pacientes muy sintomáticos.

La maniobra de acostado lateral, aunque originalmente descrita por Cohen ${ }^{16}$ como alternativa a la maniobra Dix-Hallpike (canal posterior), posteriormente se modificó al eliminar el giro de $45^{\circ}$ de la cabeza para lograr máxima estimulación de los canales laterales. Ésta se realiza sentando al paciente en el borde de la mesa de exploración y sin girar la cabeza, simplemente se acuesta sobre su costado hacia un lado; se observa la respuesta, se le regresa a la posición sentado y luego se acuesta hacia el otro lado buscando la aparición de nistagmo (Figura 5).

\section{Fisiopatología}

El mecanismo fisiopatológico que explica este cuadro clínico característico se debe a un problema «mecánico» en el oído interno, al desprenderse o dislocarse las otoconias de las máculas utricular o sacular, y depositarse en el interior de los canales semicirculares del aparato vestibular. Por el simple efecto de la gravedad, las otoconias desprendidas se alojan en el canal semicircular más declive, es decir, el canal semicircular posterior o inferior que conserva esta situación inferior, tanto con el paciente de pie como en decúbito. Es por esta razón que del 80 a $90 \%$ de los cuadros de VPPB son del canal semicircular inferior. Los otolitos desprendidos, al alojarse en los canales semicirculares, ocasionan flujos endolinfáticos inapropiados con los cambios de posición del paciente provocando corrientes de endolinfa que estimulan los sensores de movimiento angular locali-

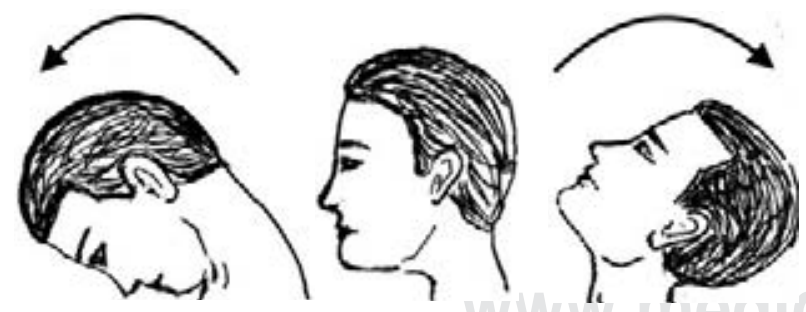

Figura 3: Maniobra de estímulo mínimo o flexión-extensión. zados en las ámpulas de cada canal.

Los otolitos pueden desprenderse y desplazarse libremente en la luz de los canales, constituyendo el mecanismo fisiopatológico conocido como canalolitia$\operatorname{sis}^{17} \mathrm{o}$ bien pueden adherirse a la cúpula en cuyo caso hablamos de una cupulolitiasis. ${ }^{18}$

En cada uno de estos casos el mecanismo fisiopatológico tiene implicaciones clínicas diagnósticas y terapéuticas. Los datos clínicos que nos ayudan a identificar una canalolitiasis son:

1. Latencia evidente (entre 10 y 20 segundos).

2. Nistagmo y vértigo menos intenso.

3. Rara vez dura más de 40 segundos.

4. Paroxismo evidente.

En contraste, una cupulolitiasis se caracteriza por:

1. Latencia muy breve (entre 5 y 10 segundos) o imperceptible.

2. Nistagmo y vértigo más intensos.

3. Puede durar más de 60 segundos.

4. Paroxismo menos evidente.

5. Presencia de micromovimiento ocular residual después del paroxismo.

Está descrito un tercer mecanismo fisiopatológico conocido como trombo o plastrón otoconial, en estos casos la cantidad de otoconias y detritus otoconiales desprendidos es tan abundante que se aglomeran formando una especie de tapón que ocluye y particiona la luz del canal semicircular afectado. ${ }^{19}$

\section{Implicaciones fisiopatológicas en los canales horizontales}

El VPPB de canal lateral es menos frecuente y explica entre el 5 a $15 \%$ de los casos, aunque algunos autores reportan una prevalencia aun mayor al $30 \% .{ }^{20}$ Debido a que ambos canales semicirculares laterales se encuentran en el mismo eje funcional con un movimiento en el plano yaw, se estimulan simultáneamente ambos lados. Aunque sólo un oído tenga otolitos dislocados, la respuesta de nistagmo se presentará indistintamente
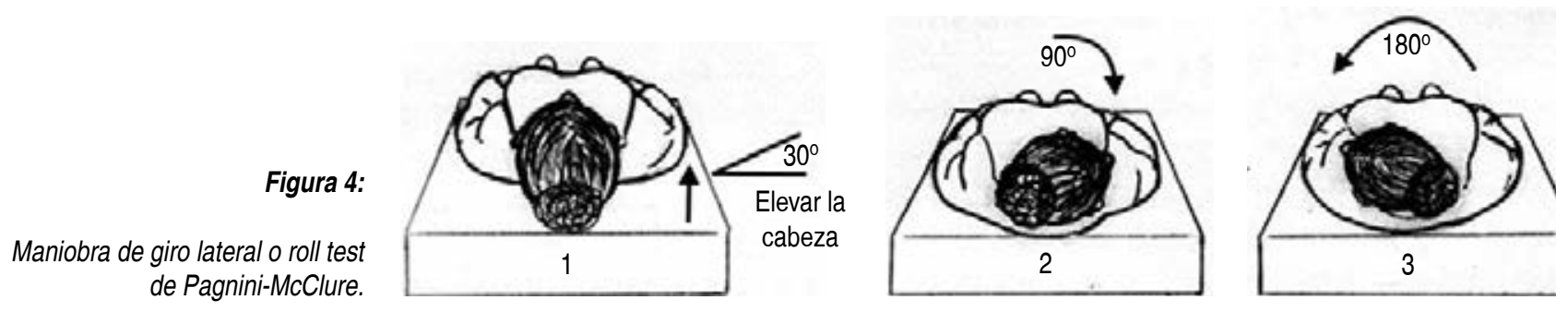


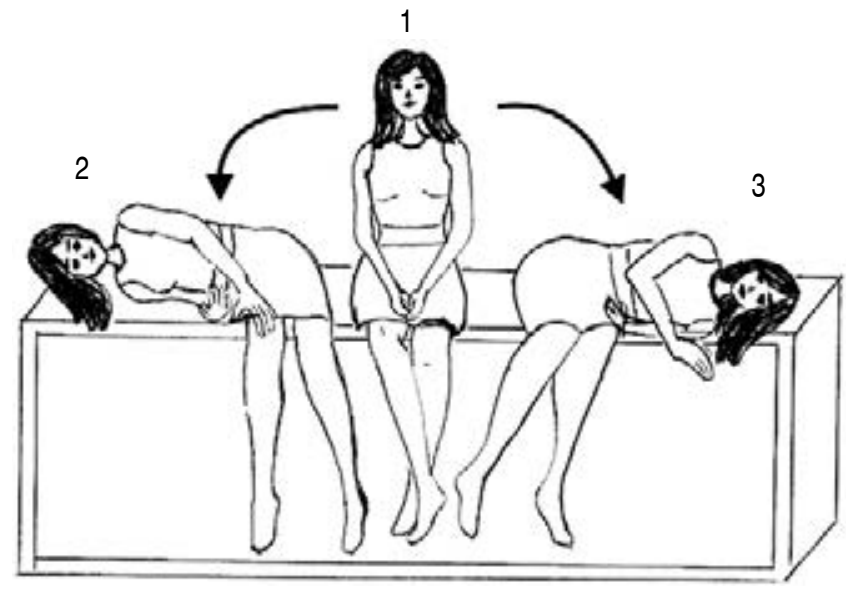

Figura 5: Maniobra de acostado lateral o side-lying.

hacia ambos lados; por esta razón, a diferencia de lo que ocurre con los canales verticales, el lado hacia donde se presenta nistagmo no indica cuál es el oído enfermo (porque siempre habrá respuesta bilateral). Para saber cuál es el lado enfermo y el mecanismo fisiopatológico en los canales laterales debemos conocer lo siguiente:

1. La dirección del nistagmo determina el mecanismo fisiopatológico. Si el nistagmo bate hacia la tierra (geotrópico) se trata de canalolitiasis. Si el nistagmo bate hacia el cielo (apogeotrópico) se trata de cupulolitiasis.

2. La intensidad del nistagmo determina el oído enfermo. En los casos de canalolitiasis (nistagmo geotrópico) el oído enfermo está hacia donde bate más intenso. En los casos de cupulolitiasis (nistagmo apogeotrópico) el oído enfermo está hacia donde bate menos intenso.

3. Las corrientes ampulípetas son excitatorias y las ampulífugas son inhibitorias. Los estímulos excitatorios siempre son más intensos que los inhibitorios (segunda ley de Ewald). ${ }^{21}$

Es muy importante determinar cuál es el oído enfermo, ya que las maniobras terapéuticas para el canal lateral por canalolitiasis siempre deben realizarse girando al paciente hacia el lado sano, de lo contrario en lugar de desalojar los otolitos dislocados los estaríamos impactando hacia el extremo ampular del canal y, por lo tanto, prolongando los síntomas. Es frecuente que los pacientes cierren los ojos cuando sienten el paroxismo del vértigo, dificultando al explorador la identificación del lado con nistagmo más intenso; en estos casos es útil preguntar al paciente de qué lado sintió más intenso el vértigo.
El estímulo de cada canal semicircular provoca un reflejo vestibulocular (VOR) característico que se debe a que cada uno de ellos está conectado a un par de músculos extraoculares, ${ }^{18,22}$ de modo tal que podemos inferir cuál es el canal afectado observando el nistagmo generado. El estímulo del canal semicircular inferior siempre generará un nistagmo mixto, hacia arriba y torsión geotrópica; el estímulo del canal semicircular superior siempre generará un nistagmo mixto, hacia abajo y en torsión apogeotrópica, y el estímulo del canal lateral siempre ocasiona nistagmo horizontal.

La nemotecnia es sencilla: nistagmo mixto que bate para arriba el canal enfermo es el contrario, es decir, el de abajo; nistagmo mixto que bate para abajo el canal enfermo es el contrario, es decir el de arriba. Es importante mencionar que el componente torsional del nistagmo está dado por los músculos oblicuos y la torsión apogeotrópica o geotrópica se refiere a que el punto de las 12 horas del iris (analogía con un reloj) bate hacia la tierra o contrario a la tierra. Si el nistagmo es horizontal puro, el canal enfermo es, por supuesto, el horizontal o lateral.

En la mayoría de los casos no se logra identificar una etiología evidente, sin embargo el interrogatorio intencionado debe indagar antecedentes de traumatismo craneano, ${ }^{11}$ neuritis vestibular, ${ }^{23}$ infecciones de vía respiratoria alta, enfermedad de Ménière, migraña vestibular, exposición a vibración mecánica intensa, cirugía otológica, implante coclear ${ }^{24}$ tratamiento odontológico o alteraciones del metabolismo del calcio. ${ }^{25}$

\section{Tratamiento}

El tratamiento del VPPB la mayoría de las ocasiones es sumamente satisfactorio tanto para el paciente como para el médico. Tomando en cuenta el

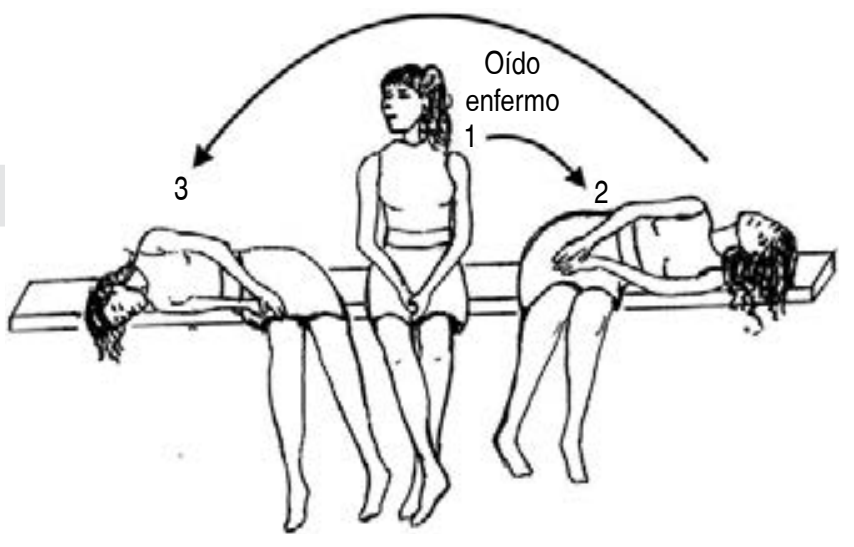

Figura 6: Maniobra de Semont. 

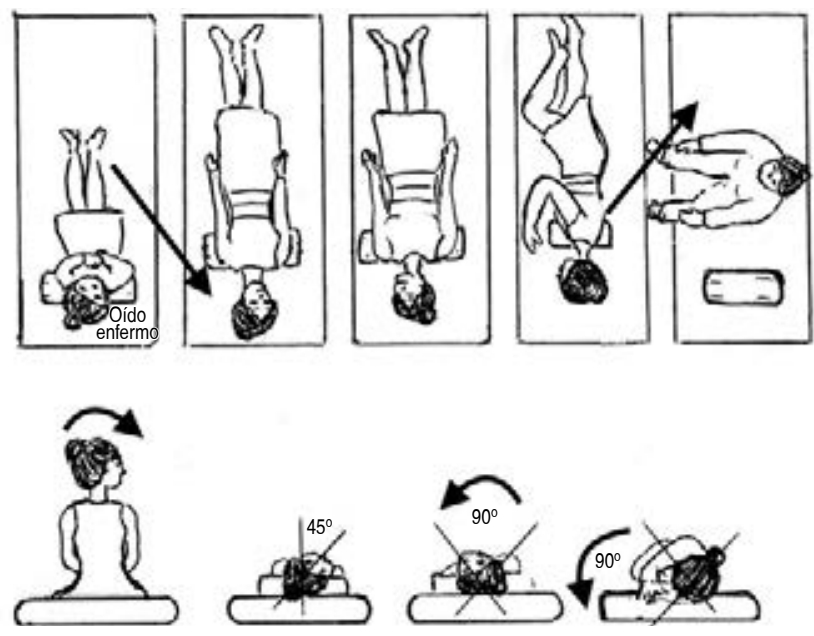

Figura 7: Maniobra de Epley.

mecanismo fisiopatológico comentado, los únicos tratamientos que elimina la sintomatología y resuelven el problema de manera inmediata son las maniobras terapéuticas o maniobras reposicionadoras o liberadoras.

\section{Maniobras terapéuticas para canales verticales}

Para tratar el VPPB de canales verticales tenemos principalmente dos maniobras: la de Alain Semont $(1988)^{26}$ y la de John Epley (1992). ${ }^{27}$

\section{Maniobra liberadora de Semont}

Se realiza con el paciente sentado en el borde de la mesa de exploración, girándole la cabeza 45 grados hacia el lado sano y acostándolo de manera lateral hacia el lado enfermo; en seguida, se realiza un movimiento con cierto impulso e intensidad para pasar rápidamente de esa posición lateral hasta el lado contrario sin girar la cabeza, de modo tal que en la primera posición la nariz queda dirigida hacia arriba, y en la segunda queda dirigida hacia abajo, porque no se modifican los $45^{\circ}$ de giro inicial hacia el lado sano (Figura 6).

\section{Maniobra liberadora de Epley}

Esta maniobra tiene los siguientes cuatro pasos: con el paciente sentado en la mesa de exploración, de manera idéntica a la maniobra diagnóstica de DixHallpike, se gira la cabeza $45^{\circ}$ hacia el lado enfermo y se acuesta al paciente, pasando a la posición de de- cúbito supino con la cabeza en ligera extensión y se espera a que ceda el nistagmo y vértigo; luego se gira la cabeza 90 grados hacia el lado contrario, en seguida se gira todo el cuerpo pasando de la posición de decúbito supino a decúbito lateral (contrario al oído enfermo), procurando no levantar ni girar la cabeza, es decir, se continúa con un giro de $45^{\circ}$ hacia el lado sano, y por último se levanta el paciente a la posición inicial de sentado sin apoyar o regresar la espalda a la mesa (Figura 7).

\section{Maniobra de Yacovino ${ }^{28}$ o cabeza colgante (head hanging)}

En el raro caso de detectar un VPPB del canal anterior, la maniobra terapéutica indicada para desplazar las otoconias del canal semicircular anterior es la de Yacovino. Ésta se realiza con el paciente sentado en la mesa de exploración como en la de Dix-Hallpike; éste se acuesta en decúbito supino, de modo tal que la cabeza sobresalga del borde de la mesa de exploración para, una vez que alcanzó la posición horizontal, continuar con una gentil hiperextensión del cuello, manteniéndolo así hasta que ceda el nistagmo-vértigo. En seguida sólo se flexiona la cabeza para pasar de hiperextensión a hiperflexión y por último se levanta al paciente nuevamente a la posición de sentado (Figura 8).

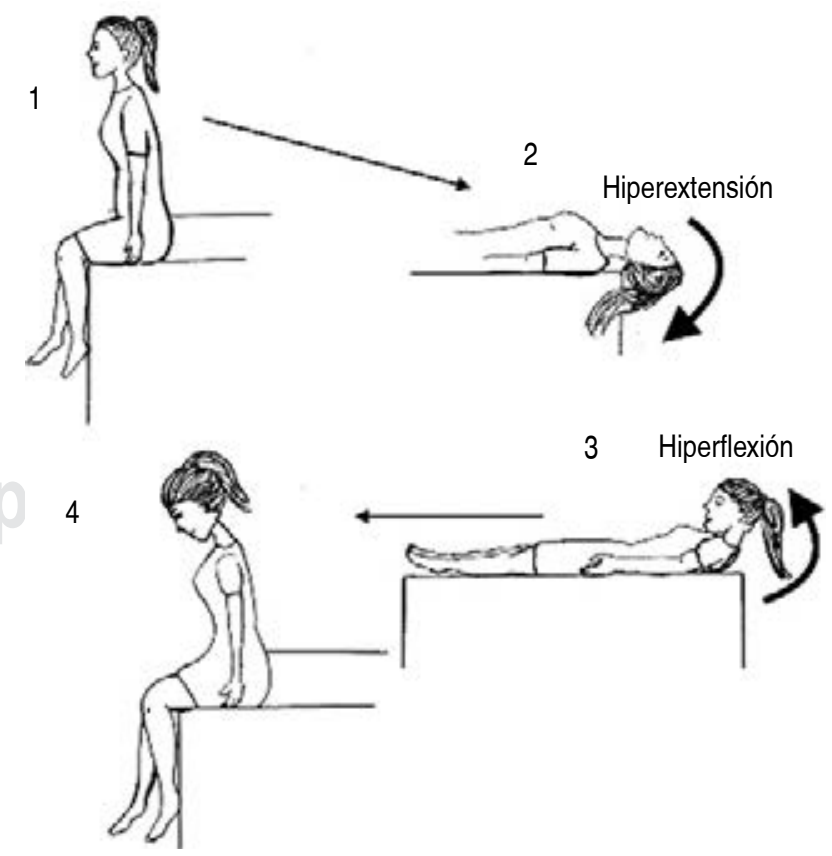

Figura 8: Maniobra de Yacovino. 


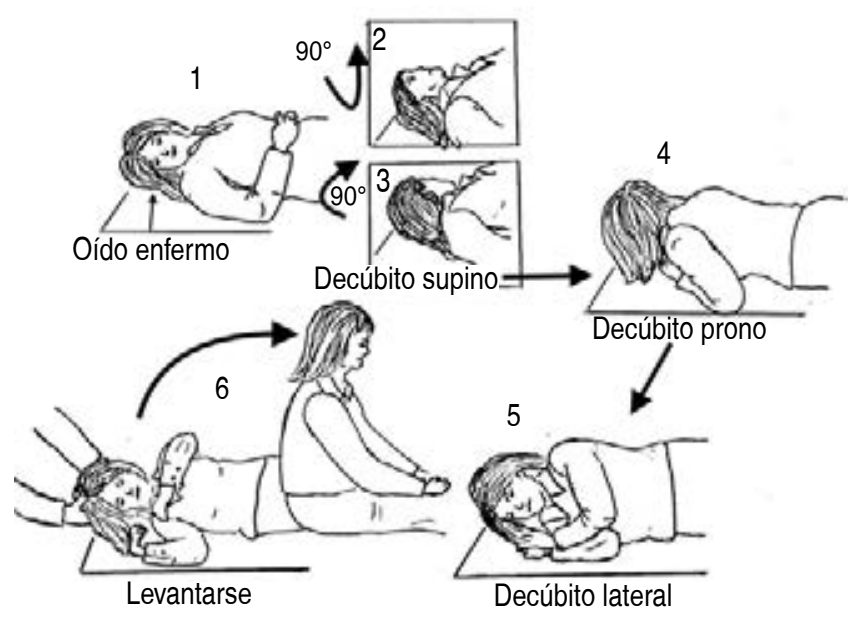

Figura 9: Maniobra de Barbecue.

\section{Maniobras terapéuticas para canales horizontales}

Para tratar adecuadamente un VPPB de canal horizontal, debemos tomar en cuanta si el mecanismo fisiopatológico es canalolitiasis o cupulolitiasis, ya que si es cupulolitiasis, primero debemos transformarla a canalolitiasis (desprender las otoconias de la cúpula) y posteriormente realizar una de las múltiples maniobras existentes, de las cuales sólo comentaremos las más conocidas.

\section{Maniobras para tratar VPPB de canal horizontal por canalolitiasis (nistagmo geotrópico): \\ Maniobra de Lempert-Tiel-Wilck o Barbecue ${ }^{29}$}

Una vez identificado el oído afectado, la maniobra se realiza en una superficie plana y blanda de preferencia; ésta inicia con el paciente en decúbito supino (la maniobra original inicia con la cara al frente, pero después se modificó girando la cara $90^{\circ}$ hacia el lado enfermo para facilitar los giros del cuerpo), se realizan tres giros rápidos de $90^{\circ}$ hacia el lado sano y se espera 30 segundos de intervalo entre cada uno de ellos hasta completar $270^{\circ}$ de giro; finalmente se regresa el paciente a la posición de sentado, obviamente el cuerpo también gira, pasando del decúbito supino a prono y luego lateral, para finalmente levantarse (Figura 9).

$$
\text { Maniobra de Gufoni }{ }^{30}
$$

En caso de canalolitiasis, al igual que todas las maniobras terapéuticas para canal horizontal, esta maniobra se realiza hacia al lado sano. El paciente se sienta en el borde de la mesa de exploración con la cabeza al frente, se acuesta lateralmente del lado no afectado y permanece en esta posición por un minuto hasta que ceda el nistagmo; en seguida la cabeza del paciente se gira rápidamente $90^{\circ}$ hacia abajo y se mantiene así por dos minutos. Finalmente regresa lentamente a la posición de sentado (Figura 10).

Maniobras para tratar VPPB de canal horizontal por cupulolitiasis (nistagmo apogeotrópico): Maniobra de Zuma ${ }^{31}$

Recientemente publicada, la maniobra de Zuma es muy útil para tratar los casos de cupulolitiasis o canalolitiasis del brazo anterior del canal, ya que este último, para efectos prácticos, se comporta como cupulolitiasis. La maniobra se lleva a cabo en cinco pasos: con el paciente sentado en el centro de la mesa de exploración, se acuesta lateralmente hacia el lado enfermo esperando en esta posición tres minutos; en seguida sube sus piernas a la mesa de exploración y gira su cabeza y cuerpo $90^{\circ}$ hacia arriba quedando en decúbito supino y esperando otros tres minutos; a continuación gira su cabeza $90^{\circ}$ hacia el lado sano y espera en esta posición otros tres minutos, y después flexiona su cabeza hacia adelante. Por último, se regresa a la posición de sentado manteniendo la cabeza flexionada y girada como la tenía (Figura 11).

\section{Maniobra de Gufoni modificada (invertida y extendida)}

Con el apoyo de un modelo tridimensional del oído interno que permite visualizar los movimientos necesarios para corregir una cupulolitiasis del canal horizontal, hemos ideado una maniobra que ha sido útil en el tratamiento exitoso de pacientes con esta enfermedad. Consiste en realizar una maniobra de Gufoni invertida, es decir, hacia el lado enfermo en

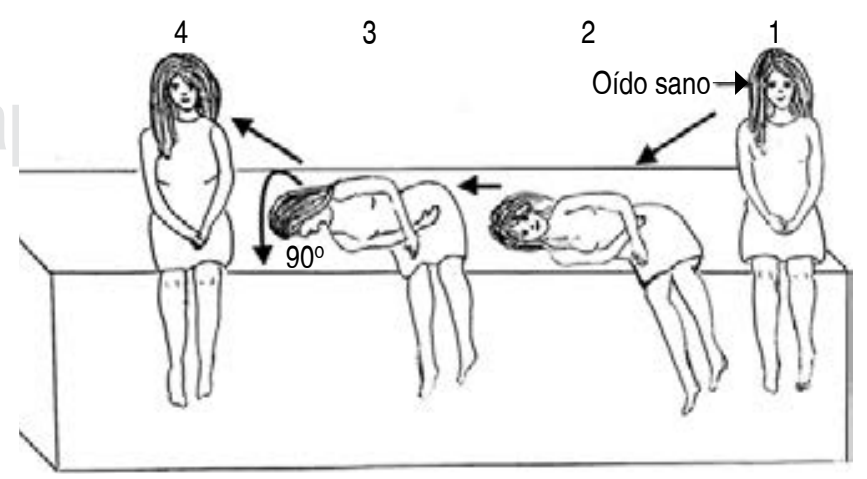

Figura 10: Maniobra de Gufoni. 


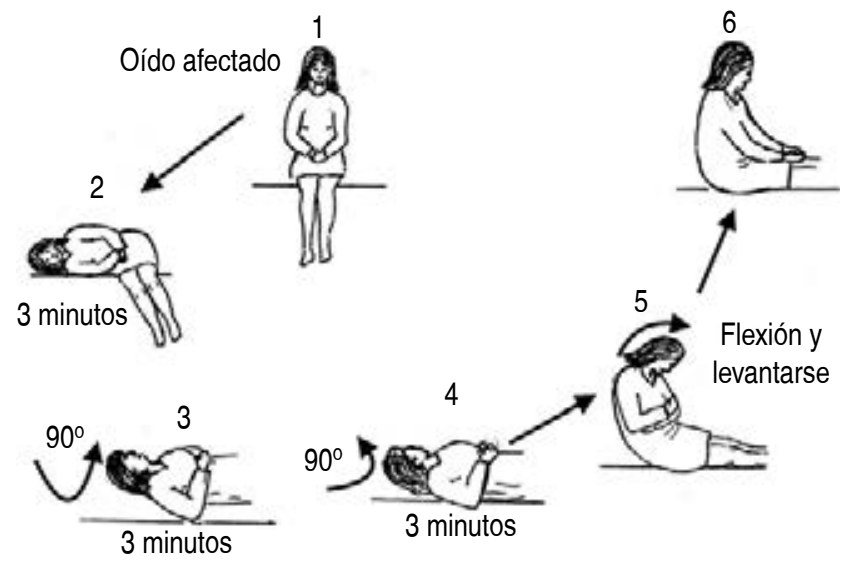

Figura 11: Maniobra de Zuma.

lugar de hacia el sano y girar la cabeza hacia arriba en lugar de hacia abajo y extendida, porque se continúa el movimiento con dos giros de $90^{\circ}$ hacia el lado sano hasta completar $270^{\circ}$. Se realiza en cuatro pasos: con el paciente sentado en el centro de la mesa de exploración, éste se acuesta lateralmente hacia el lado enfermo y se espera dos minutos para desprender las otoconias de la cúpula y transformar una cupulolitiasis en canalolitiasis; en seguida gira su cabeza $90^{\circ}$ hacia arriba y espera otros dos minutos; a continuación sube sus piernas a la mesa de exploración para girar y quedar en decúbito supino y gira su cabeza otros $90^{\circ}$ hacia el lado sano al menos un minuto; por último realiza otro giro de $90^{\circ}$ de la cabeza y el cuerpo para quedar en decúbito lateral con la nariz hacia abajo. Es importante que permanezca al menos dos minutos en las dos primeras posiciones para que, por inercia y gravedad, las otoconias de la cúpula se desprendan (se puede usar un vibrador para favorecer la dislocación de las otoconias adheridas a la cúpula) al menos un minuto en las siguientes posiciones (Figura 12).

\section{Comentarios finales}

Es importante comentar que la mayoría de los pacientes que tuvieron un cuadro de VPPB una vez que éste ha cedido ya sea de manera espontánea o gracias a la intervención del médico al realizar una maniobra terapéutica, ${ }^{32}$ quedan con un malestar residual que corresponde al síntoma de mareo, definido como una sensación perturbada o alterada de la orientación espacial sin sensación falsa o distorsionada de movimiento. ${ }^{1}$ Es posible que este síntoma residual se deba a disfunción utricular ${ }^{33} \mathrm{y}$ es necesario comentarle al paciente que una vez que haya desaparecido el vértigo, definido como sensación de movimiento propio cuando no ocurre ningún movimiento propio o la sensación distorsionada de movimiento propio durante un movimiento normal de la cabeza, ${ }^{1}$ el mareo puede permanecer por algunos días o incluso semanas. En algunos pacientes el mareo es tan intenso que limita sus actividades cotidianas y es cuando se justifica el tratamiento farmacológico como coadyuvante para el síntoma residual, una vez que ha cedido el vértigo, aunque también pueden usarse medicamentos para disminuir los síntomas vasovagales y la ansiedad en caso de que estos últimos sean muy severos.

En realidad, el tratamiento con las maniobras liberadoras, aunque es muy efectivo sólo es paliativo, ya que un tratamiento etiológico sería el que resuelva la causa por la cual los otolitos se desprenden de las máculas utricular y sacular.

Estudios recientes han intentado abordar este problema desde un punto de vista etiológico y parece ser que existen mecanismos alterados en el metabolismo del calcio y vitamina $\mathrm{D}^{34}$ que provocan modificaciones bioquímicas en la red trabecular gelatinosa que sostiene los otolitos, y también alteraciones en la producción de otoconias que modifican sus cualidades iónicas, alterando su adhesividad y favoreciendo su dislocación y desplazamiento hacia los canales semicirculares; esto explicaría el alto índice de recurrencia observado (hasta 50\%) y la preponderancia del sexo femenino en relación a las alteraciones hormonales relacionadas con la osteopenia y osteoporosis que las hacen más propensas a padecer VPPB. ${ }^{35}$

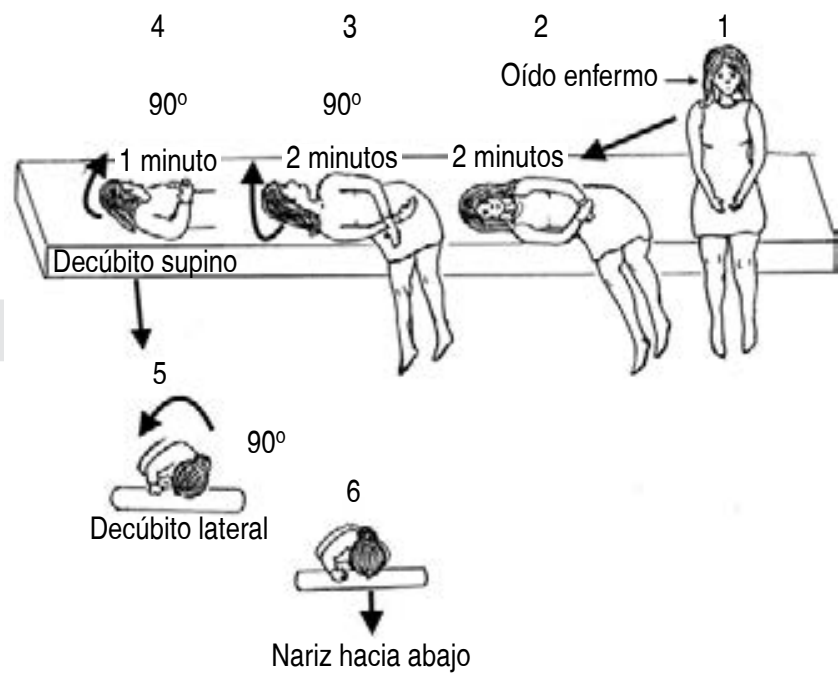

Figura 12: Maniobra de Gufoni modificada (invertida y extendida). 


\section{BIBLIOGRAFÍA}

1. Bisdorff A, Von Brevern M, Lempert T, Newman-Toker DE. Classification of vestibular symptoms: towards an international classification of vestibular disorders. J Vestib Res. 2009; 19 (1-2): 1-13.

2. Pollak L, Klein C, Rafael S, Vera K, Rabey JM. Anxiety in the first attack of vertigo. Otolaryngol Head Neck Surg. 2003; 128 (6): 829-834

3. Pratt RT, McKenzie W. Anxiety states following vestibular disorders. Lancet. 1958; 2 (7042): 347-349.

4. Hanley K, O'Dowd T, Considine N. A systematic review of vertigo in primary care. Br J Gen Pract. 2001; 51 (469): 666-671.

5. Neuhauser HK, von Brevern M, Radtke A, Lezius F, Feldmann M, Ziese T et al. Epidemiology of vestibular vertigo: a neurotologic survey of the general population. Neurology. 2005; 65 (6): 898-904

6. Parnes LS, Agrawal SK, Atlas J. Diagnosis and management of benign paroxysmal positional vertigo (BPPV). CMAJ. 2003; 169 (7): 681-693.

7. Bhattacharyya N, Gubbels SP, Schwartz SR, Edlow JA, ElKashlan H, Fife T et al. Clinical practice guideline: benign paroxysmal positional vertigo (update). Otolaryngol Head Neck Surg. 2017; 156 (3_suppl): S1-S47.

8. von Brevern M, Radtke A, Lezius F, Feldmann M, Ziese T, Lempert $\mathrm{T}$ et al. Epidemiology of benign paroxysmal positional vertigo: a population based study. J Neurol Neurosurg Psychiatry. 2007; 78 (7): 710-715.

9. Nunez RA, Cass SP, Furman JM. Short- and long-term outcomes of canalith repositioning for benign paroxysmal positional vertigo. Otolaryngol Head Neck Surg. 2000; 122 (5): 647-652.

10. Bloom J, Katsarkas A. Paroxysmal positional vertigo in the elderly. J Otolaryngol. 1989; 18 (3): 96-98.

11. Baloh RW, Honrubia V, Jacobson K. Benign positional vertigo: clinical and oculographic features in 240 cases. Neurology. 1987; 37 (3): 371-378.

12. Dix MR, Hallpike CS. The pathology symptomatology and diagnosis of certain common disorders of the vestibular system. Proc R Soc Med. 1952; 45 (6): 341-354.

13. Jackson LE, Morgan B, Fletcher JC Jr, Krueger WW. Anterior canal benign paroxysmal positional vertigo: an underappreciated entity. Otol Neurotol. 2007; 28 (2): 218-222.

14. Yetiser S, Ince D. Diagnostic role of head-bending and lyingdown tests in lateral canal benign paroxysmal positional vertigo. Otol Neurotol. 2015; 36 (7): 1231-1237.

15. Pagnini P, Nuti D, Vannucchi P. Benign paroxysmal vertigo of the horizontal canal. ORL J Otorhinolaryngol Relat Spec. 1989; 51 (3): 161-170.

16. Cohen HS. Side-lying as an alternative to the Dix-Hallpike test of the posterior canal. Otol Neurotol. 2004; 25 (2): 130-134.

17. Brandt T, Steddin S. Current view of the mechanism of benign paroxysmal positioning vertigo: cupulolithiasis or canalolithiasis? J Vestib Res. 1993; 3 (4): 373-382.

18. Lechner C, Taylor RL, Todd C, Macdougall H, Yavor R, Halmagyi GM et al. Causes and characteristics of horizontal positional nystagmus. J Neurol. 2014; 261 (5): 1009-1017.
19. Jackler RK, Brackmann DE. Neurotology. Chapter 41: Benign paroxysmal positional vertigo. 2nd ed. St Louis: Mosby; 2005. pp. 644-658.

20. Nuti D, Mandalà M, Salerni L. Lateral canal paroxysmal positional vertigo revisited. Ann N Y Acad Sci. 2009; 1164: 316-323.

21. Carey JP, Della-Santina CC. Chapter 163: Principles of applied vestibular physiology. In: Flint PW, Haughey BH, Lund VJ, Niparko JK, Richardson MA, Robbins JR et al. Cummings otolaryngology-head and neck surgery. 5th ed. Philadelphia, PA: Elsevier; 2015. pp. 2510-2514.

22. Simpson JI Graf W. The selection of reference frames by nature and its investigators. In: Berthoz A Melville-Jones G, eds. Adaptive mechanisms in gaze control: facts and theories. Amsterdam: Elsevier Science Publishers; 1985. pp. 3-16.

23. Harada K, Oda M, Yamamoto M, Nomura T, Ohbayashi S, Kitsuda C. A clinical observation of benign paroxysmal positional vertigo (BPPV) after vestibular neuronitis (VN). Acta Otolaryngol Suppl. 1993; 503: 61-63.

24. Viccaro M, Mancini P, La Gamma R, De Seta E, Covelli E, Filipo R. Positional vertigo and cochlear implantation. Otol Neurotol. 2007; 28 (6): 764-767.

25. Jeong SH, Kim JS. Impaired calcium metabolism in benign paroxysmal positional vertigo: a topical review. J Neurol Phys Ther. 2019; 43 Suppl 2 Supplement, Special Supplement: International Conference on Vestibular Rehabilitation: S37-S41.

26. Semont A, Freyss G, Vitte E. Curing the BPPV with a liberatory maneuver. Adv Otorhinolaryngol. 1988; 42: 290-293.

27. Epley JM. The canalith repositioning procedure: for treatment of benign paroxysmal positional vertigo. Otolaryngol Head Neck Surg. 1992; 107 (3): 399-404.

28. Yacovino DA, Hain TC, Gualtieri F. New therapeutic maneuver for anterior canal benign paroxysmal positional vertigo. J Neurol. 2009; 256 (11): 1851-1855.

29. Lempert T, Tiel-Wilck K. A positional maneuver for treatment of horizontal-canal benign positional vertigo. Laryngoscope. 1996; 106 (4): 476-478.

30. Gufoni M, Mastrosimone L, Di Nasso F. Repositioning maneuver in benign paroxysmal vertigo of horizontal semicircular canal. Acta Otorhinolaryngol Ital. 1998; 18 (6): 363-367.

31. Zuma e Maia F. New treatment strategy for apogeotropic horizontal canal benign paroxysmal positional vertigo. Audiol Res. 2016; 6 (2): 163.

32. Sekine K, Imai T, Sato G, Ito M, Takeda N. Natural history of benign paroxysmal positional vertigo and efficacy of Epley and Lempert maneuvers. Otolaryngol Head Neck Surg. 2006; 135 (4): 529-533.

33. von Brevern M, Schmidt T, Schönfeld U, Lempert T, Clarke $\mathrm{AH}$. Utricular dysfunction in patients with benign paroxysmal positional vertigo. Otol Neurotol. 2006; 27 (1): 92-96.

34. Talaat HS, Abuhadied G, Talaat AS, Abdelaal MS. Low bone mineral density and vitamin $\mathrm{D}$ deficiency in patients with benign positional paroxysmal vertigo. Eur Arch Otorhinolaryngol. 2015; 272 (9): 2249-2253.

35. Yu S, Liu F, Cheng Z, Wang Q. Association between osteoporosis and benign paroxysmal positional vertigo: a systematic review. BMC Neurol. 2014; 14: 110. 